\title{
On the characterization of magnetic reconnection in global MHD simulations
}

\author{
T. V. Laitinen ${ }^{1}$, P. Janhunen ${ }^{2,1}$, T. I. Pulkkinen ${ }^{1}$, M. Palmroth ${ }^{1}$, and H. E. J. Koskinen ${ }^{1,2}$ \\ ${ }^{1}$ Finnish Meteorological Institute, Space Research, PL 503, 00101 Helsinki, Finland \\ ${ }^{2}$ University of Helsinki, Department of Physical Sciences, PL 64, 00014 Helsinki, Finland
}

Received: 29 March 2006 - Revised: 17 August 2006 - Accepted: 14 September 2006 - Published: 22 November 2006

\begin{abstract}
The conventional definition of reconnection rate as the electric field parallel to an $\mathrm{x}$-line is problematic in global MHD simulations for several reasons: the $\mathrm{x}$-line itself may be hard to find in a non-trivial geometry such as at the magnetopause, and the lack of realistic resistivity modelling leaves us without reliable non-convective electric field. In this article we describe reconnection characterization methods that avoid those problems and are practical to apply in global MHD simulations. We propose that the reconnection separator line can be identified as the region where magnetic field lines of different topological properties meet, rather than by local considerations. The global convection associated with reconnection is then quantified by calculating the transfer of mass, energy or magnetic field across the boundary of closed and open field line regions. The extent of the diffusion region is determined from the destruction of electromagnetic energy, given by the divergence of the Poynting vector. Integrals of this energy conversion provide a way to estimate the total reconnection efficiency.
\end{abstract}

Keywords. Magnetospheric physics (Instruments and techniques) - Space plasma physics (Magnetic reconnection; $\mathrm{Nu}$ merical simulation studies)

\section{Introduction}

A little over 50 years ago Dungey (1953) introduced the idea that magnetic "lines of force can be broken and rejoined". Since then the concept of magnetic reconnection has found important applications in diverse space environments. Dungey (1961) himself applied it to the terrestrial magnetosphere, sketching the basic magnetospheric convection pattern with reconnection occurring on the magnetopause and

Correspondence to: T. V. Laitinen

(tiera.laitinen@fmi.fi) in the tail. Study of solar flares prompted Sweet (1958) and Parker (1957) to develop the first quantitative reconnection model. Petschek (1964) then invented a model that could explain the fast energy release rates observed in the solar eruptions. Reconnection is also a somewhat disputed ingredient in dynamo theories (for a review see Kulsrud, 1999) that aim to explain the magnetic fields of stars and galaxies.

All of the abovementioned phenomena, and several others where reconnection may play a role, are nowadays modelled using magnetohydrodynamic (MHD) simulations. Reconnection has been shown to occur in, for example, global simulations of the magnetosphere (e.g. Walker et al., 1993; Fedder et al., 1995; Raeder, 1999; Laitinen et al., 2005). However, quantitative analyses of reconnection processes in MHD simulations and of their significance to the phenomena being simulated are difficult, because theoretical characterizations of reconnection are often impractical or even impossible to apply. We will discuss this in Sect. 3.

Reconnection has been studied quite extensively by devoted simulations. For example, the GEM magnetic reconnection challenge (Birn et al., 2001, and references therein) compared reconnection rates in different types of simulation codes under standard boundary conditions. These "theoretical simulations" are useful for learning about the reconnection process itself and about the capabilities of different simulation schemes, but they often seem to be more reminiscent of theoretical considerations than practically oriented simulations. Our aim in this article is to develop methods for studying reconnection as a part of a larger, more selfcontained physical process, methods that work in large-scale MHD simulations. We focus on the magnetosphere, but we expect that some of the ideas presented here might prove useful also in other applications of plasma physics.

To test and illustrate the methods presented here, we use the Gumics-4 ideal MHD code (Janhunen, 1996, introduces the previous version Gumics-3; version 4 is described in more detail for example in Janhunen and Palmroth, 2001, and

Published by Copernicus GmbH on behalf of the European Geosciences Union. 


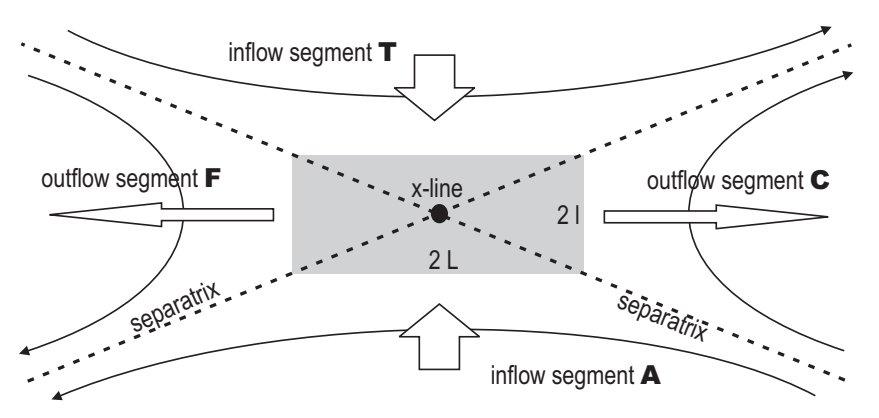

Fig. 1. Simple reconnection at an $x$-line, or a separator. The four separatrices that meet at the separator divide the space into four topologically distinct segments, which are labeled as $\mathrm{F}($ ree $)$, $\mathrm{T}$ (oward), A(way) and $\mathrm{C}$ (losed) for comparison with Fig. 2. The shaded area represents the diffusion region $D_{R}$, and its dimensions are referred to as $2 L \times 2 l$ when the Sweet-Parker model is discussed in Sect. 6.

Palmroth et al., 2001). It is a global three-dimensional simulation of the coupled solar wind-magnetosphere-ionosphere system. The magnetospheric part, which is of interest in this study, solves the ideal MHD equations in their conservative form. There is no explicit resistivity; diffusion in the simulation is produced by the numerical solution. The code applies the finite volume method where quantities are treated as cell averages, and uses the Roe solver to solve the Riemann problem of calculating fluxes of conserved quantities through the cell interfaces (LeVeque, 1992). In the rare cases when the Roe method produces intermediate states with negative pressure the Harten-Lax-van Leer (HLL) solver is used (Janhunen, 2000). Elliptic cleaning is used to maintain $\nabla \cdot \boldsymbol{B}=0$, since this is not otherwise guaranteed by the finite volume method. The grid in Gumics-4 is an adaptive Cartesian octogrid with semiautomatic refinement. While running, the time-accurate code splits and recombines grid cells using steepness of gradients as the spatial resolution refinement criterion. In diffusion regions gradients are strong and accordingly the cell size turns out to be the smallest allowed, which is $0.25 R_{E}$.

Sections 2 and 3 of this paper are of introductory nature: we discuss briefly the three-dimensionalization of the familiar two-dimensional reconnection problem, global MHD simulation schemes, and the interplay of resistivity, numerical diffusion and parallel electric fields. In Sect. 4 we introduce a classification of magnetospheric field lines by their topological properties and derive thereof a separator line, certain parts of which can be associated with the reconnection $\mathrm{x}$-line. In Sect. 5 we describe how to utilise the field line classification to produce quantitative estimates of the transfer of mass, energy and magnetic field. Finally, in Sect. 6 we examine the energy conversion property of reconnection making use of the Poynting vector and its divergence.

\section{Problems with the concept of $x$-line in three dimen- sions}

In two dimensions reconnection occurs at an x-point. The plane around the $\mathrm{x}$-point is divided into four topologically distinct segments by four separatrices; magnetic field lines are brought towards the x-point in two opposite segments, reconnected at the $\mathrm{x}$-point and then transported away in the two other segments (Fig. 1). Reconnection thus allows, and is always associated with, flow of plasma across the separatrices. In a static case the flow pattern implies an electric field perpendicular to the plane. As shown by Vasyliunas (1975), this electric field is proportional to the reconnection rate, that is, the amount of magnetic flux reconnected per time interval. The outflow segments are usually narrow and contain a current sheet. This configuration can be extended into three dimensions by assuming translational invariance in the third direction. The x-point then becomes an x-line, also known as the separator, having an electric field parallel to it.

An ideal setting for this kind of essentially twodimensional reconnection in nature can be found between the tail lobes of the magnetosphere. There the central surface of the current sheet can be determined by locating the magnetic field direction change or the current density maximum. Then the $x$-line is just the sign change location of the magnetic field component normal to this surface. This simple method has been applied and illustrated in Laitinen et al. (2005) and is not treated here.

In a fully three-dimensional setting, where the reconnecting fields need not be antiparallel and symmetries need not be present, an $\mathrm{x}$-like field geometry alone is not a sufficient indicator of reconnection: a simple example provided by Hesse and Schindler (1988) is the field $\left(B_{x}, B_{y}, B_{z}\right)=(y, x, 1)$, where any given point $\left(x_{0}, y_{0}, z_{0}\right)$ is an $\mathrm{x}$-point in a suitably oriented plane and thus any field line would geometrically be an $\mathrm{x}$-line. Furthermore they showed that in this configuration plasma can flow across the apparent "separatrices", although there is no electric field component along the "separator" and field lines are conserved. This is because there are no topologically distinct field regions and thus no real separatrices in the configuration even though two-dimensional cuts so suggest. This example shows that a reconnection $\mathrm{x}$-line cannot in general be identified by considering only the local magnetic field geometry and plasma flow. In Sect. 4 we describe how this problem can be avoided in magnetospheric context by basing the separator definition on global properties of field lines, without reference to local field geometry. A similar definition could be applied also in other environments where the field lines in some of the segments in Fig. 1 connect to a nearby surface. Solar corona and tokamaks are examples of such environments. 


\section{$3 E_{\|}$and its problems in MHD}

Several authors (e.g. Axford, 1984; Schindler et al., 1988) avoid the problems of defining separatrices in three dimensions by defining reconnection simply as "localized breakdown of the frozen-in field condition". Schindler et al. (1988) showed that such "general magnetic reconnection" has global effects if and only if it is associated with a parallel electric field, more precisely, if and only if

$$
\int \boldsymbol{E}_{\|} d s \neq 0
$$

on a measurable set of field lines in a limited diffusion region $D_{R}$. The integral is to be taken along a field line within $D_{R}$. On the other hand, Priest and Forbes (2000) prefer to adopt a more restrictive definition of reconnection which essentially requires a field line with a parallel electric field and an $\mathrm{x}$-like magnetic field geometry on the perpendicular plane.

As the significance of $E_{\|}$is so unanimously acknowledged, why not use it as the reconnection proxy in MHD simulations? Electric field is often not calculated by the actual MHD solver. It is a post-processing variable calculated afterwards from the primary variables through the Ohm's law,

$\boldsymbol{E}=-\boldsymbol{v} \times \boldsymbol{B}+\boldsymbol{R}$.

Here $\boldsymbol{R}$ is a general non-idealness term. A necessary and sufficient condition for the existence of a parallel electric field is that $\boldsymbol{R}$ be non-zero and not perpendicular to $\boldsymbol{B}$. In nature $\boldsymbol{R}$ can have several contributions from, for example, collisions, fluctuations, and particle inertia. When the concept of reconnection is applied, it is assumed that $\boldsymbol{R}$ is non-zero in a limited diffusion region $D_{R}$ and (approximately) vanishes elsewhere so that field-line conservation holds outside the diffusion region.

MHD simulations have different approaches to determining $\boldsymbol{R}$. The simplest one is ideal MHD, which is also the choice taken in Gumics-4: $\boldsymbol{R} \equiv 0$. This implies through Eq. (2) that there is no parallel electric field. $\boldsymbol{E}$ either vanishes at the separator, if $\boldsymbol{B}$ or $\boldsymbol{v}$ vanishes, or otherwise is perpendicular to the separator (which is a field line). Ideal MHD simulations thus do not produce the reconnection electric field.

Reconnection in ideal MHD models is made possible by numerical diffusion. This in turn depends on the discretization scheme and numerical solver used, and is difficult to quantify. Discretization and numerical diffusion may also produce local small-scale discrepancies at such special locations as the surroundings of a separator, where $\boldsymbol{v}$ and $\boldsymbol{B}$ change appreciably over the size of a grid cell. As an example, at the magnetotail reconnection site in Gumics the magnetic $x$-line and the plasma flow reversal line are usually separated by a distance of several grid cells. This is discussed more closely by Laitinen et al. (2005). What is relevant here is that the electric field calculated from Eq. (2) with $\boldsymbol{R}=0$ becomes reversed in a small region between the $\mathrm{x}$-line and the flow reversal line. This result is a consequence of the numerical method chosen for this calculation and is independent of the grid size. Thus calculating the electric field along or near the separator is clearly out of the question, since that is just the place where it is not modelled correctly.

Depending on the procedure for calculating $\boldsymbol{E}$ from the primary variables, some $E_{\|}$may be produced by purely numerical effects as explained by Siscoe et al. (2001). While this numerical $E_{\|}$naturally maximizes in regions where $v$ and $\boldsymbol{B}$ have large gradients, such as at reconnection sites, it is in our opinion not evident that it should be straightforwardly associated with the physical $E_{\|}$of a reconnection process.

In the magnetosphere, the plasma density is sufficiently low to make the classical collisional resistivity effectively zero. Thus, any resistive effects arise from other processes such as wave-particle interactions or turbulence. The form of the resistivity and its dependence on the local parameters is largely an open issue. However, in resistive MHD electric resistance is modelled explicitly by inserting $\boldsymbol{R}=\eta \boldsymbol{J}$ into Ohm's law. Here $\eta$ is the resistivity and is usually either assumed constant or made to depend on the current density $\boldsymbol{J}$ in a step function-like manner (Raeder, 1999).

In resistive MHD a physically meaningful electric field can be produced even in the diffusion region of a reconnection process. $E_{\|}$may then be applicable as a proxy for reconnection. However, the problematic numerical effects discussed above in the context of ideal MHD are present in the resistive approach as well, and it may be difficult to determine whether the total electric field is physically realistic even at the reconnection site where numerical contributions are large. Furthermore, $\eta$ has to be chosen on a somewhat ad hoc basis, and the use of different $\eta$ 's in different runs and different simulation codes complicates comparisons.

Hall effects are not included in basic MHD, but the Hall term $\boldsymbol{R}=\boldsymbol{J} \times \boldsymbol{B} / n e$ is sometimes added to model them. Because it is of second order in $\boldsymbol{B}$ (since $\mu_{0} \boldsymbol{J}=\nabla \times \boldsymbol{B}$ ), it renders the system of equations non-hyperbolic. This makes the use of Godunov techniques problematic, but the Hall effect can be added to numerical solvers as a correction term. Hall effects are observed in magnetospheric reconnection (Øieroset et al., 2001; Vaivads et al., 2004) and the GEM simulation challenge (Birn et al., 2001, and references therein) showed that they have an important effect on MHD reconnection rate under standard boundary conditions. However, the Hall term is perpendicular to $\boldsymbol{B}$ and thus does not directly produce $E_{\|}$.

The methods described hereafter aim to be independent of the exact simulation scheme. Their applicability does not depend on what kind of non-idealness term $\boldsymbol{R}$ is assumed in the Ohm's law. 


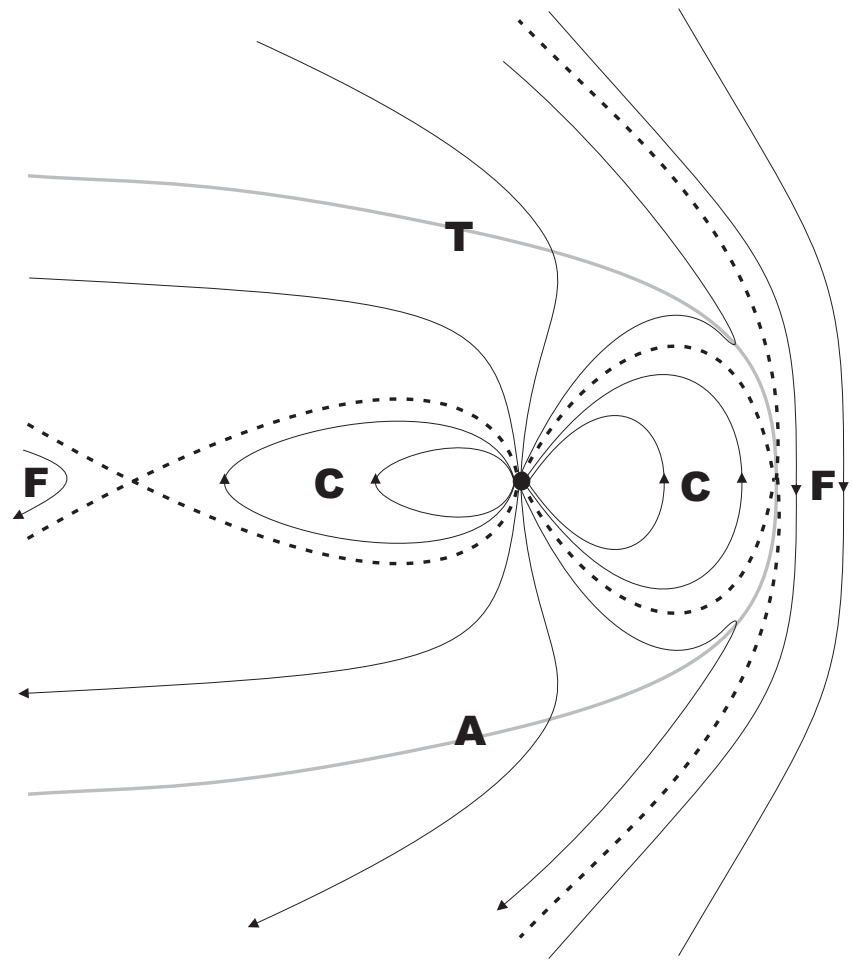

Fig. 2. The four topologically different magnetic field regions in the magnetosphere during southward IMF. T stands for toward the Earth, A for away, $\mathrm{C}$ for closed and $\mathrm{F}$ for free field lines. The figure is drawn for southward IMF, and under these conditions the four regions are seen to meet at the magnetopause and magnetotail reconnection sites. Figure 3 will show that the separator drapes around the Earth along the flanks of the magnetopause. Compare also with Figs. 1a, b, 2a and 4a in Park et al. (2006).

\section{The four-field junction}

As discussed in Sect. 2, the x-line can unfortunately be found reliably by local considerations only in few special situations where the the relationship between local geometry and global topology is extraordinarily evident. This led us to consider searching for the separator as the "meeting place" of magnetic field lines with different global properties. Qualitatively the separator line was described in this sense by Cowley (1973). While such a global definition is not useful in local reconnection theory and is problematic to generalize, in space applications it is exactly the global consequences of reconnection processes that are of most interest, and therefore it is useful to characterize reconnection by its global properties.

As shown by Fig. 1, separator is the line where four separate regions of magnetic field meet. Figure 2 illustrates how these four regions can be identified by the global properties of their field lines in the terrestrial magnetosphere. The field lines can be classified in four classes:
1. Free, or disconnected. These are solar wind field lines not attached to the Earth.

2. Away. These are open field lines whose "back end" is attached to the Earth. They emanate from a region of northern magnetic polarity. In the magnetosphere the southern tail lobe is formed by away-type field lines.

3. Toward. Open field lines whose "front end" is attached to the Earth. They emanate from a region of southern polarity and form the northern tail lobe.

4. Closed. Attached to the Earth from both ends.

Configurations of closed and open field lines at the magnetopause reconnection region in a global MHD simulation have recently been examined and illustrated by Park et al. (2006).

In a global MHD simulation the junction of these four regions of magnetic field can be found in the following manner. First, set up a Cartesian grid with spacing $\delta$ in a region where a separator line is expected to exist. Starting from each grid point, trace the magnetic field both forwards and backwards, that is, integrate the field line. If the tracing encounters the Earth in some direction, that end of the field line is called attached; otherwise, i.e. if the integration ends at the outer boundary of the simulation box or continues for a prespecified maximum length without encountering a boundary, that end of the field line is said to be open. Then, if both ends of the field line are open, the grid point is classified as free. If the forward end is open (attached) and the backward end attached (open), the grid point is of away-type (towardtype). And if both ends are attached, the grid point is closed. Finally, loop through the grid once more: if within a distance $\Delta$ from a given point lies grid points of all four categories, then the point under inspection belongs to the set of fourfield junction points. In practice the field line integration is the computationally heavy part of this procedure, so it is wise to save the classification of grid points as an intermediate result.

The final result is strictly speaking not a line but a set of points. However, these points tend to form a line or ribbon as illustrated in Fig. 3. The figure was produced from a test run on Gumics-4 with slowly (10 degrees every $10 \mathrm{~min}$ ) rotating interplanetary magnetic field (IMF). At the time depicted the field was pointing dawnward. The IMF magnitude was 10 $\mathrm{nT}$, solar wind speed $400 \mathrm{~km} / \mathrm{s}$, density $7.47 \mathrm{~cm}^{-3}$ and temperature $1 \times 10^{5} \mathrm{~K}$. The above-mentioned parameters of the four-field junction search were $\delta=0.25 R_{E}$ and $\Delta=3 \delta$.

The separator line in Fig. 3 crosses the subsolar magnetopause in a tilted orientation, which depends on the IMF clock angle $\theta=\arctan \left(B_{y} / B_{z}\right)$. Along the flanks of the magnetopause the line reaches tailward beyond $x=-20 R_{E}$. It crosses the tail between the tail lobes and thus forms a loop around the Earth. This is consistent with the qualitative picture presented by Cowley (1973) for southward IMF. The two 
gaps in the cross-tail part are caused by the coarseness of the grid used: the $\mathrm{x}$-configuration in the tail is so thin that no nearby grid points happened to touch the closed and open segments (compare Fig. 1) in those parts of the tail. This also happens sometimes at the nose of the magnetopause. Very small IMF clock angles $\left(|\theta| \lesssim 30^{\circ}\right)$ are also problematic for the four-field junction technique: northern and southern cusp reconnection create toward- and away-type field lines which cross the nose region adjacent to each other and create fourfield junction points there, although there is no reconnectionrelated separator line at the nose. Otherwise the four-field junction is a stable and well-behaved representation of the separator line, and it also evolves smoothly in time as solar wind conditions change.

When IMF acquires a large positive $Z$ component the separator line becomes genuinely discontinuous: the crosstail part recedes away or disappears when tail reconnection ceases, and also the subsolar part disappears when the IMF clock angle is small enough, that is, when the IMF is strongly northward. The behaviour of the separator line as a function of the IMF clock angle will be analyzed more closely in a subsequent paper.

The green line in Fig. 3 is the x-line of tail reconnection found independently by considering the local geometry of the field. The current sheet centre was first determined from $B_{x}$ sign change, and then the x-line from $B_{n}=0$, where $B_{n}$ is the magnetic field component normal to the current sheet (Laitinen et al., 2005). The geometrical method was thus designed to find an $\mathrm{x}$-line that is roughly perpendicular to the $\mathrm{x}$-axis, which may explain why the agreement is very good in the central part of the tail $\mathrm{x}$-line but not so good in the tailward-receding side parts.

Finally it must be stressed that the sole existence of a fourfield junction does not imply that reconnection would be taking place there. The four-field junction represents a separator line in the global topology of the magnetosphere, and by itself tells nothing about the dynamics. Only after consideration of other signatures of reconnection, such as plasma flow pattern or Poynting vector divergence, it may be justifiable to interpret some parts of the separator line as reconnection $\mathrm{x}$-lines. Also, small-scale reconnection may occur elsewhere; an example of this are the so-called flux transfer events, which are probably caused by small sporadic reconnection events (Russell and Elphic, 1978; Fedder et al., 2002; Sonnerup et al., 2004). They do not normally involve field lines of all the four topological categories defined above, and hence are not revealed by the four-field junction technique. Similarly, tail reconnection escapes the four-field junction technique if it occurs on closed field lines; when it advances to lobe field lines, the $\mathrm{x}$-line becomes a topological separator in the sense required by the method, and the line is detected.

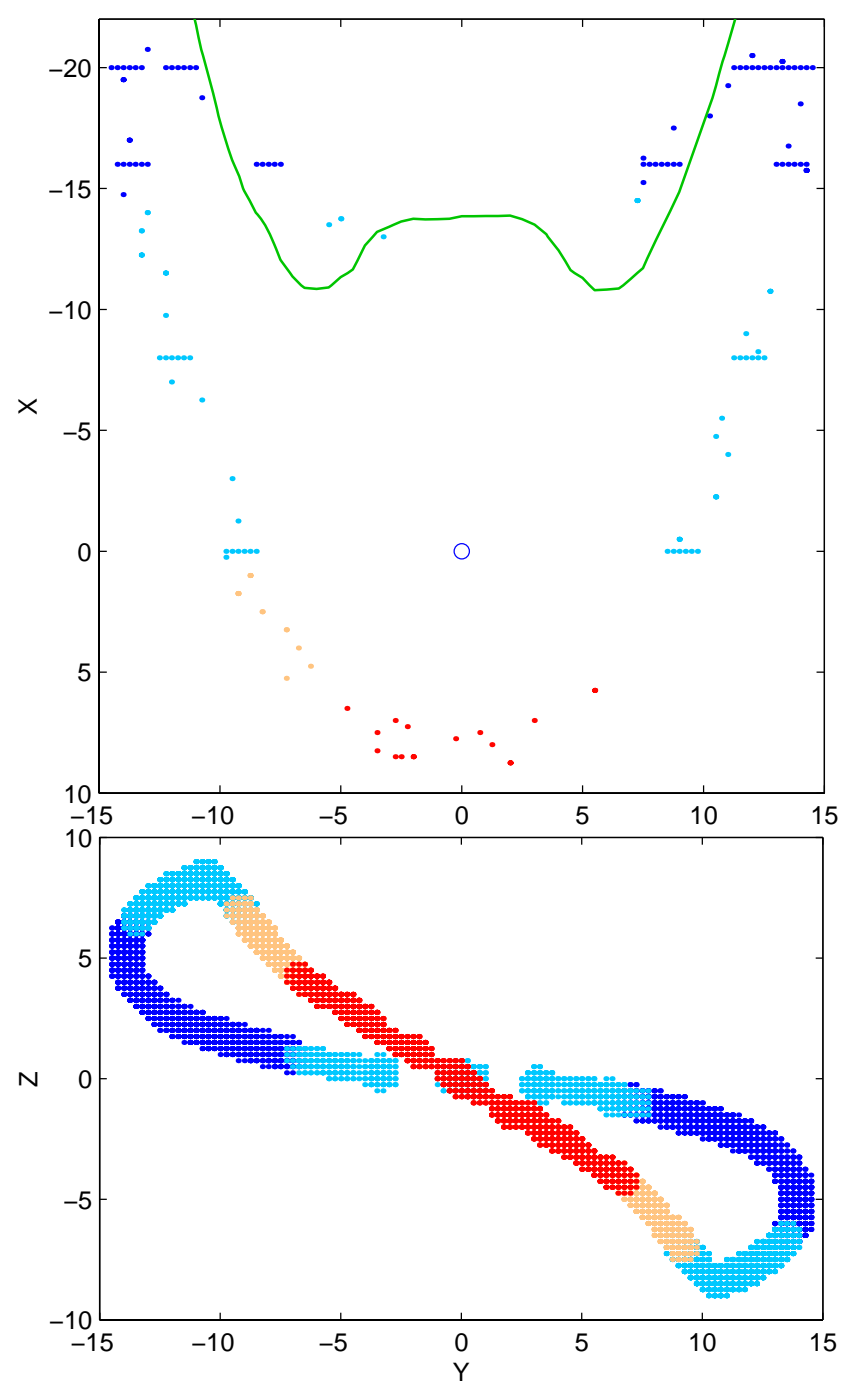

Fig. 3. The four-field junction in Gumics-4, seen from north (upper panel) and from the Sun (lower panel). The points are coloured by their $X$ coordinate to aid in identifying different parts of the junction in the lower panel. The green line is the magnetotail $x$-line given by an independent search procedure based on the local field geometry.

\section{Topological transfer quantities}

The classification of grid points as belonging to either free, toward, away, or closed magnetic domain, introduced in the previous section, can also be used to measure quantitatively the transfer of mass and energy from one topological domain to another. In the following toward-type, away-type, and free field lines are collectively called open.

The procedure is as follows: Choose a domain of interest, say, the closed field line region. The points of the grid are now interpreted as the centres of cubic cells. Go through the grid cells $i$ and for each closed cell, check also the status of the six neighbouring cells $j$. When an open cell $j$ is found, 

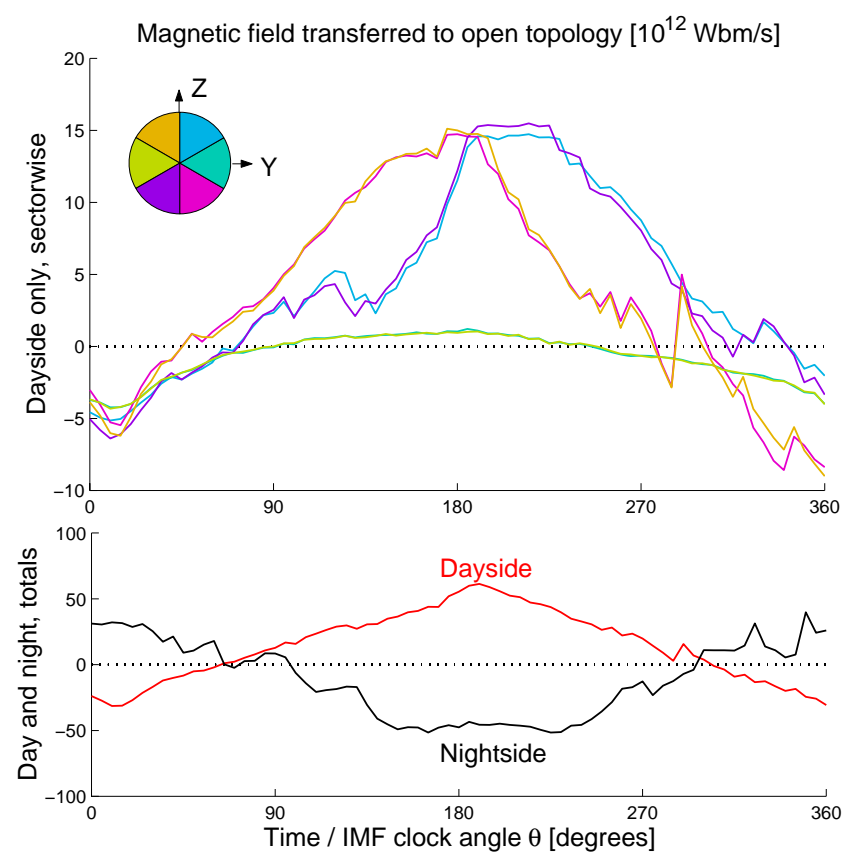

Fig. 4. The amount of magnetic field transferred from topologically closed to other regions. In the top panel, the transfer is calculated on the dayside $(X>0)$ in six sectors; the colour-coding of the sectors is illustrated by a small front view of the magnetopause. In the lower panel the total amount of opening magnetic field in the dayside and the nightside are compared.

let $\hat{\boldsymbol{n}}$ be a unit vector in the direction from $i$ to $j$. The mass flow from cell $i$ to cell $j$ is then $\delta T_{m}=\rho \boldsymbol{v} \cdot \hat{\boldsymbol{n}} A$, where density $\rho$ and velocity $\boldsymbol{v}$ are evaluated at the cell interface between $i$ and $j$, and $A$ is the area of that interface. Summing these contributions from all closed-open cell pairs gives the total mass flux out of the domain of closed field lines. This is a discretization of the integral

$T_{m}=\int_{S} \rho \boldsymbol{v} \cdot d \boldsymbol{S}$,

where $S$ is the outer surface of the closed field line region, or some chosen part of that surface.

This analysis assumes that the topological configuration is quasi-stationary, in other words, that the boundary of closed and open field lines moves only very slowly compared to plasma velocities. This approximation may not always be valid if for example the magnetosphere is compressed by a sudden pressure pulse in the solar wind, but we will not treat this question here.

Mass transfer is of course not the only transfer quantity that can be computed. Replacing $\rho v$ with the total energy flux $\boldsymbol{K}$ leads to the energy transfer $T_{E}$. An interesting quantity would be the rate of opening or closure of magnetic flux, that is, the reconnection voltage. Analoguously with the mass transfer, the amount of magnetic field transferred across the closed-open field line boundary is

$T_{B}=\int_{S}\left|\boldsymbol{B}_{S}\right| \boldsymbol{v} \cdot d \boldsymbol{S}$,

where $\boldsymbol{B}_{S}$ is the component of $\boldsymbol{B}$ parallel to the surface $S$. However, the dimensionality of this quantity is not a voltage but $\mathrm{Tm}^{3} / \mathrm{s}=\mathrm{Wbm} / \mathrm{s}=\mathrm{Vm}$. This magnetic transfer could thus be described as "the sum of the lengths of magnetic field lines cut open by reconnection", whereas the reconnection voltage would simply be "the number of field lines cut open by reconnection". To arrive at a voltage, one would need to calculate the transfer of magnetic field not through a surface but across a curve, and there is no natural way to choose such a curve from a three-dimensional simulation space. But despite being of unconventional dimensionality, the magnetic transfer can convey interesting information on magnetopause reconnection as demonstrated by Fig. 4 .

Figure 4 is based on a simulation run where the IMF direction rotated through one full rotation in the $Y Z$-plane while the IMF magnitude and all other solar wind parameters stayed constant. The full rotation took six hours, IMF clock angle changing $10^{\circ}$ every $10 \mathrm{~min}$. The time that the tail reconnection takes to settle to a new steady state after a change in IMF is $20-30 \mathrm{~min}$, so the figure must not be read as a sequence of steady states, but the IMF rotation is slow enough to allow comparison of dusk-, south- and dawnward IMF's, for example. The horizontal axis represents time but is labeled with clock angle in degrees for clarity.

The upper panel in the figure shows transfer of magnetic field from closed to open field lines in the dayside $(X>0)$ only. The domain of integration is subdivided into six sectors, which are colour-coded as illustrated by the inserted small front view of the magnetosphere. Opposite sectors show nearly identical behaviour; even many transient fluctuations such as those around $\theta=270^{\circ}$ in the red and orange curves are similar. This indicates that the calculation method is robust and describes global processes, not local fluctuations.

Figure 4 further shows that during southward IMF magnetic flux opens preferentially in those sectors that lie along the IMF direction in the $Y Z$-plane. In the low-latitude sectors magnetic transfer is very small. This is consistent with Palmroth et al. (2003), who report similar sector-dependence for energy flow through the magnetopause in the Gumics simulation. In the lower panel of the same figure the red curve is obtained by summing the six curves of the upper panel, and the black curve is the corresponding total magnetic field opening rate in the nightside $(X<0)$. The curves roughly sum to zero, which is not surprising, as it only means that during southward IMF the field lines that open on the dayside close in the tail. During strongly northward IMF the signs are opposite and interpretation is not as straightforward, but high-latitude reconnection probably produces new closed field lines on the dayside. 
The mass, energy or magnetic transfer through any topological boundary could be calculated similarly, but in the magnetospheric context the open-closed boundary seems to be the most natural choice because it is the only one that forms a closed surface. Thus the flux can be integrated over the whole surface, whereas on the other boundary surfaces the integration area would have to be selected arbitrarily. On the other hand, arbitrary dividing of the integration surface can be informative as was seen in Fig. 4.

\section{Poynting vector divergence}

We now turn from the topological properties of reconnection to its other central feature: energy conversion. Reconnection is invoked as a means to rapidly release magnetic energy in, for instance, the near-Earth neutral line model of magnetospheric substorms (Baker et al., 1996). Qualitatively, Papadopoulos et al. (1999) discuss the energy conversion location by mapping Poynting vector flow lines from the solar wind through the magnetopause and showing how they focus in the inner magnetotail to the tail reconnection site. A more quantitative tool to measure the rate at which electromagnetic energy is converted into other forms (in a steady state, when $\left.\partial_{t}=0\right)$ is the divergence of the Poynting vector, $\nabla \cdot S$.

\subsection{Energy conversion in Sweet-Parker model}

As an introduction to the subject we calculate $\nabla \cdot S$ for the Sweet-Parker reconnection. In this most basic of reconnection models magnetic annihilation and plasma acceleration take place in an elongated diffusion region betweeen two regions of antiparallel magnetic fields. Following the notation of Priest and Forbes (2000, pp. 120-123), let the length of the diffusion region be $2 L$ and thickness $2 l$ (see Fig. 1). Subscripts $i$ and $o$ refer to the inflow and outflow regions outside the diffusion region, respectively. It can be shown that the inflow velocity is

$v_{i}=v_{A i} / \sqrt{\mathcal{R}_{m i}}$

and the outflow velocity $v_{o}=v_{A i}$, where $v_{A i}$ is the inflow Alfvén speed and $\mathcal{R}_{m i}$ is the magnetic Reynolds number based on the inflow Alfvén speed.

Generally speaking, the Reynolds number is a measure of the relative importance of magnetic convection versus diffusion. If diffusion is caused by scalar resistivity $\eta$, the magnetic Reynolds number can be estimated by $\mathcal{R}_{m}=\mu_{0} L V_{0} / \eta$, where $V_{0}$ is the "typical" speed scale of the system. When calculated using the Alfvén speed, like above, where $\mathcal{R}_{m i}=\mu_{0} L v_{A i} / \eta$, it is also known as the Lundquist number. But as the "typical" speed scale here is set by the outflow velocity which equals the inflow Alfvén speed, this number can be identified as the global magnetic Reynolds number, and so we will call it simply the magnetic Reynolds number and drop the subscript $i$.
The thickness of the diffusion region is determined by

$l=L / \sqrt{\mathcal{R}_{m}}$,

and the magnitude of the Poynting vector towards the diffusion region is

$S_{i}=\frac{E_{i} B_{i}}{\mu_{0}}=\frac{v_{i} B_{i}^{2}}{\mu_{0}}$.

In the outflow regions the density of Poynting flux away from the diffusion region is

$S_{o}=\frac{1}{\mu_{0}} v_{o} B_{o}^{2}=\frac{1}{\mu_{0}} \frac{L}{l} v_{i}\left(\frac{l}{L} B_{i}\right)^{2}=\frac{l}{L} S_{i}$.

As it is assumed that $l \ll L$, the outgoing Poynting flux can be neglected in this order-of-magnitude estimate. The consumption of Poynting flux in the diffusion region is then $\nabla \cdot S=-\left(2 S_{i}\right) /(2 l)$. Combining this with Eqs. (5-7) leads to

$\nabla \cdot S=-\frac{v_{A i} B_{i}^{2}}{\mu_{0} L}$.

It is interesting to note that this result is independent of $\mathcal{R}_{m}$ and that the maximal value of $-\nabla \cdot \boldsymbol{S}$ in simulated reconnection should thus not depend on numerical diffusion, if the reconnection is of Sweet-Parker nature. However, the thickness of the diffusion region does depend on $\mathcal{R}_{m}$ (Eq. 6) and therefore the reconnection process as a whole is of course not independent of diffusion.

Figure 5 shows $\nabla \cdot S$ in the magnetotail reconnection region together with magnetic field lines. The figures are drawn from a simulation run where the solar wind dynamic pressure was $8 \mathrm{nPa}$ (from $v=400 \mathrm{~km} / \mathrm{s}$, density $=13.3 \mathrm{~cm}^{-3}$ ) and magnetic field strength $10 \mathrm{nT}$. The IMF direction rotated slowly and is indicated in the figure for each panel. A Sweet-Parker-like Poynting flux sink region appears at the same time when the magnetic field geometry undergoes a qualitative transformation into an x-type configuration (panels a and $\mathrm{b}$ ). As the driving of the reconnection grows stronger, the Poynting sink intensifies. In panel (c), $-\nabla \cdot S$ peaks at a little over $10^{-10} \mathrm{~W} / \mathrm{m}^{3}$. At the same time in the tail lobes outside the diffusion region typical numbers are $B=40 \mathrm{nT}$ and $v_{A}=3000 \mathrm{~km} / \mathrm{s}$, which is also the maximal tailward outflow velocity. The half-length of the diffusion region, or the global length scale, is about $L=5 R_{E}$. Inserting these into Eq. (9) gives $-\nabla \cdot S=1.2 \times 10^{-10} \mathrm{~W} / \mathrm{m}^{3}$. Equation (9) gives good estimates to the upper limit of $-\nabla \cdot \boldsymbol{S}$ of tail reconnection also in other simulation runs with somewhat different numbers.

6.2 A proxy for the spatial distribution of reconnection efficiency on a surface

In panels (b) and (c) of Fig. 5a narrow white stripe appears in the middle of the blue diffusion region. This is most likely a 

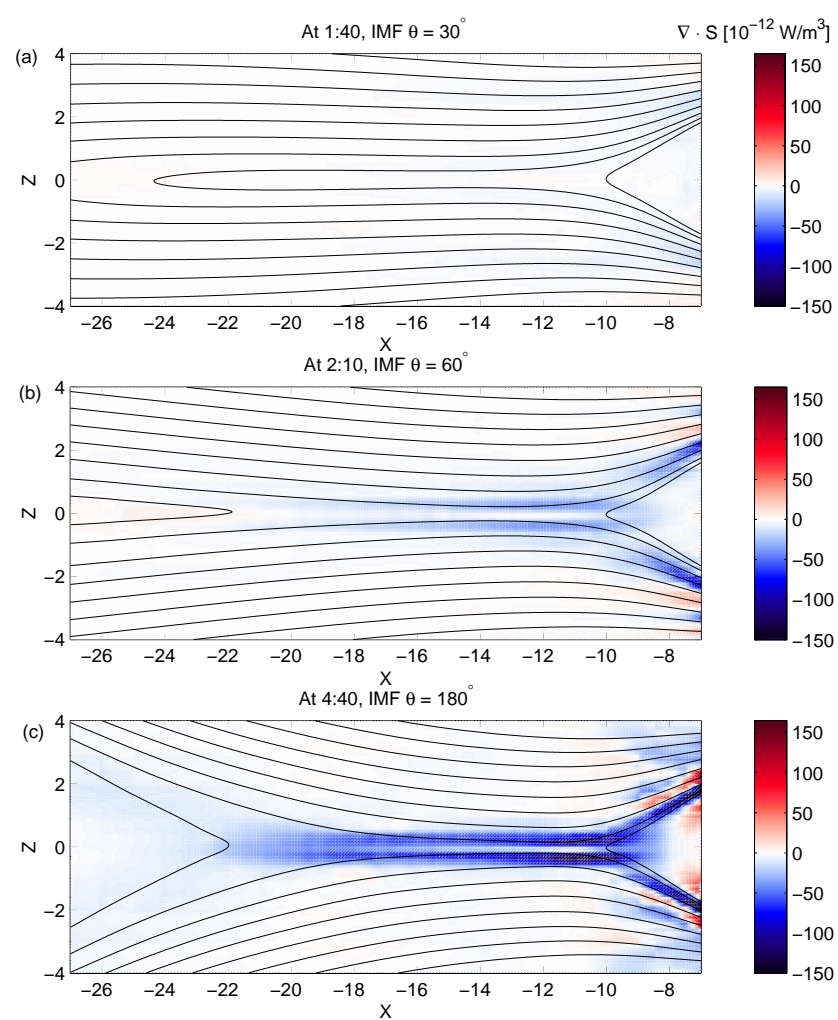

Fig. 5. The magnetotail reconnection region depicted on an $X Z$ plane. Black lines represent magnetic field and colouring shows the Poynting divergence $\nabla \cdot S$. In blue areas electromagnetic energy is transformed into mechanical energy. (a) Reconnection has not yet started. (b) Weak and (c) strong reconnection.

numerical feature having no physical relevance. At the magnetopause we also observe a layered structure, but there is no symmetry across the diffusion region and $\nabla \cdot S$ sometimes attains even positive values. What if we tried to determine the extent of the diffusion region by plotting $\nabla \cdot S$ on the tail current sheet or on the magnetopause? The tail current sheet intersects the $X Z$-plane of Fig. 5 exactly in the white stripe, wherefore the result would be spectacularly erroneous. Similarly the result on the magnetopause would be fatally sensitive to small variations in determining the magnetopause surface location.

An obvious solution to this problem is to integrate out the layers. Laitinen et al. (2005) quantified the total energy conversion rate of tail reconnection by defining the reconnection power as

$P_{\mathrm{rec}}=-\oint_{\partial V} \boldsymbol{S} \cdot \hat{\boldsymbol{n}} d a$,

where the volume $V$ is to encompass the whole reconnection region. (By Gauß's theorem this is equivalent to volume integral of $\nabla \cdot S$.) But what if the extent of reconnection region is not known from other considerations? If reconnection can be expected to occur on a known surface, such as the tail current sheet or the magnetopause, then one may define energy conversion surface density by integrating $\nabla \cdot S$ along the normal of that surface over a suitably chosen interval $\left[-l_{1}, l_{2}\right]$ :

$\sigma_{\mathrm{Ec}}=-\int_{-l_{1}}^{l_{2}} \nabla \cdot S d l$.

In the Sweet-Parker model this is, by Eqs. (6) and (9), and setting $l_{1}=l_{2}=l$,

$\sigma_{\mathrm{Ec}}=\frac{2 v_{A i} B_{i}^{2}}{\mu_{0} \sqrt{\mathcal{R}_{m}}}$.

This equation can also be solved for $\mathcal{R}_{m}$ to give an estimate of the numerical diffusion in a simulation, since all other quantities can be calculated from the simulation results. When applied to the tail reconnection in Gumics, Eq. (12) gives the order-of-magnitude result $\mathcal{R}_{m} \sim 100$. This estimate has to be considered with some caution, as the use of Eq. (12) is based on the assumption that Sweet-Parker-merging is occurring at its maximal rate. That assumption predicts the local value of $\nabla \cdot S$ correctly. However, the Sweet-Parker model is based on resistive MHD while reconnection in the ideal MHD simulation is allowed by numerical diffusion; the two phenomena appear very similar, but it is not necessarily meaningful to identify them with each other in every aspect.

Two side remarks on the energy conversion surface density are in order. First, although its integral is the reconnection power (Eq. 10), we prefer not to use the word "reconnection" when referring to $\sigma_{\mathrm{Ec}}$. That word should be reserved for processes that change the connectivity of field lines, while energy conversion can take place in other ways, for example, as simple diffusion. Second, Eq. (11) neglects the curvature of the surface on which $\sigma_{\mathrm{Ec}}$ is calculated. A more correct formulation would weigh more the values on the convex side of the surface and less those on the concave side, such that the integral of $\sigma_{\mathrm{Ec}}$ over some surface area would equal the volume integral of $\boldsymbol{\nabla} \cdot \boldsymbol{S}$ over a volume obtained by inflating that surface area into a sheet. The error in the approximation of Eq. (11) is of the order of $\max \left(l_{1}, l_{2}\right) / R$, where $R$ is the smallest curvature radius of the surface at the given point. The curvature radius of the magnetopause is $R \gtrsim 10 R_{E}$ and we usually use $l_{1}=l_{2}=1.5 R_{E}$, so the computationally simple Eq. (11) is well applicable to produce surface maps. Volume integrals are more robustly done without the intermediate step of $\sigma_{\mathrm{Ec}}$.

Figure 6 illustrates the usability of energy conversion surface density by showing it on the magnetopause under four different IMF orientations. Solar wind dynamic pressure was $2 \mathrm{nPa}$ and IMF magnitude $10 \mathrm{nT}$. (This is the same run that was used for Fig. 3.) The integration range of Eq. (11) was $1.5 R_{E}$ to both directions from the magnetopause surface, which was determined using plasma flow lines as defined by Palmroth et al. (2003). The figure reveals that during southward IMF magnetic energy is consumed in a wide region 
around the subsolar point. Behind the polar cusps there are also regions that produce magnetic energy; magnetopause reconnection is thus associated with considerable dynamo action. All this supports the view that at least in MHD reconnection is a large-scale process that cannot be described exhaustively by inspecting only the $\mathrm{x}$-line or its surroundings.

\subsection{Generalization beyond specific reconnection models}

The previous discussion is based on the simple Sweet-Parker model. That is well suited for Gumics, where reconnection appears as a large diffusion region. This seems to be the case also for other global MHD codes: for example, Dorelli et al. (2004) describe the reconnection produced in the GGCM (Geospace General Circulation Model) global MHD simulation as occurring in a macroscopic current sheet "rather than in Petschek-like configurations". On the other hand, there are observational evidence of Petschek-like slow mode shocks in the magnetotail (e.g. Ho et al., 1994; Eriksson et al., 2004), and also some hybrid tail simulations report Petschek configurations (Krauss-Varban and Omidi, 1995). It is thus an interesting question to what extent the Sweet-Parker-based discussion on Poynting vector divergence can be generalized.

The Petschek reconnection model and its many more advanced or generalized variants (Priest and Forbes, 2000) still contain a small Sweet-Parker-like diffusion region which allows the actual re-connection of field lines. The discussion in Sect. 6.1 can still be applied to this diffusion region, with the caveat that $L$ is now only the size of the diffusion region and not a global length scale. The magnetic Reynolds number $\mathcal{R}_{m}$, which has $L$ in its definition, also is no more globally meaningful.

However, from the global point of view the energy budget of the diffusion region is not very interesting when most of the energy processing takes place on shock fronts outside it. A more fruitful approach for a global simulator is to analyze the energetics of the whole reconnection process. The reconnection power and energy conversion surface density (Eqs. 10 and 11) are suited exactly to this purpose, since their definitions contain no assumptions on the nature of the processes causing the energy conversion. Equation (12) is specific to the Sweet-Parker-model, but similar predictions could be given for different reconnection models using their respective theoretically predicted inflow speeds. Otherwise the discussion in Sect. 6.2 is model-independent.

When applying these Poynting-derived proxies to Petschek-type reconnection, for example, the integration domains must be chosen so that they contain all the shock fronts. If the shock pairs have a relatively small opening angle, $\sigma_{\mathrm{Ec}}$ still describes informatively the distribution of energy conversion on a surface. It does not tell the difference between a diffusion region and a shock pair; in order to assess the three-dimensional structure of the reconnection, one can in addition examine the distribution of $\nabla \cdot \boldsymbol{S}$ on a surface perpendicular to the neutral line, as is done in Fig. 5.
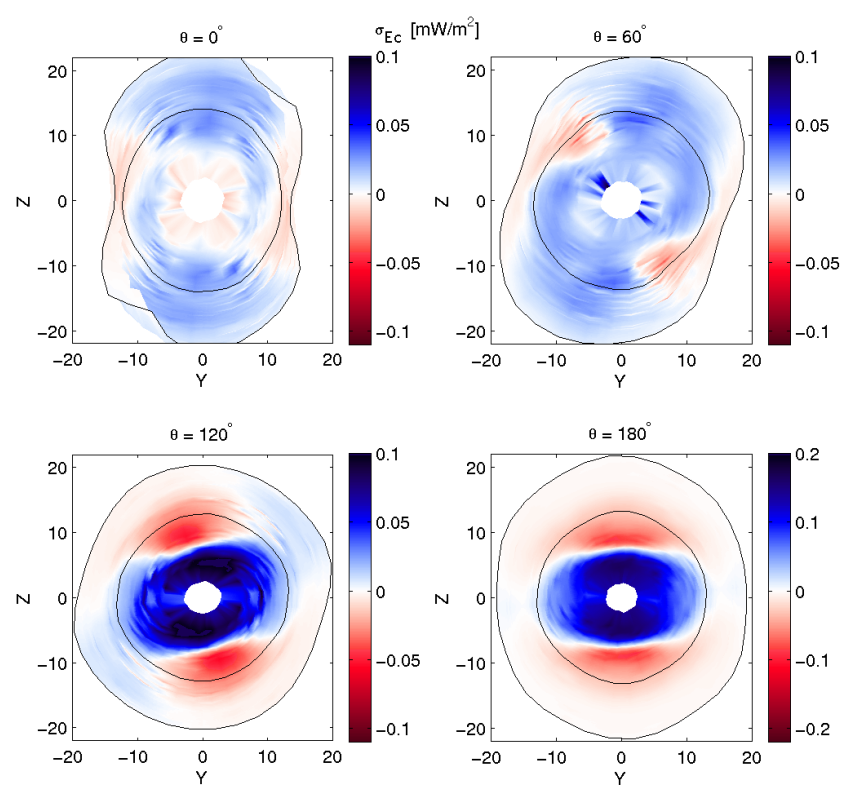

Fig. 6. Energy conversion surface density on the magnetopause under four different IMF orientations. The IMF direction is shown by an arrow and also given in degrees above each panel. The lines are intersections of the magnetopause with the planes $X=0$ and $X=-30$. The white hole at the subsolar point is a technical feature caused by the plotting procedure. Note the different colour scale in the lower-right panel!

In practice one also needs to do this to determine suitable integration limits to use in definition (11).

\section{Discussion and conclusions}

For a long time, reconnection has been known to be the key process in energy coupling between the solar wind and the magnetosphere (e.g. Baker et al., 1996). However, observing the reconnection process poses significant challenges, as the key processes occur at a very small-scale diffusion region, while the consequences of the diffusion region dynamics occur over a wide region of space. While for example the Cluster measurements have brought significant new information about processes near the diffusion region (e.g. Øieroset et al., 2001; Vaivads et al., 2004), the large-scale effects of reconnection can only be inferred using empirical proxies or from large-scale simulation results (e.g. Pulkkinen et al., 2006). For improved understanding of the nature of the solar windmagnetosphere coupling it is important to develop quantitative methods to analyze the locations and large-scale effects of reconnection in the simulation, such as those discussed in this paper.

We have described practical approaches to characterizing magnetic reconnection in global MHD simulations: The four-field junction is a way to define the separator line as 
a meeting place of magnetic field lines with different topological properties; this definition works well on the magnetopause where the complicated geometry makes it difficult to find an $\mathrm{x}$-line by local considerations. However, the fourfield junction alone is not sufficient to characterize reconnection. Strong conversion of magnetic energy, evidenced by negative divergence of the Poynting vector, in a region traversed by a separator line, is already a good indicator of reconnection. The divergence of the Poynting vector provides an estimate of reconnection efficiency locally and can also be integrated to give the reconnection power, that is, total rate of energy conversion by reconnection. The global convection associated with reconnection can be estimated from the transfer of mass, energy or magnetic field across the boundaries of topologically separate magnetic domains.

The Gumics-4 simulation produces two reconnection sites that fulfill the criteria of a separator line and energy conversion. They are large diffusion regions that lie in the tail and at the magnetopause. Energy conversion takes place in a region vastly larger than the separator line, which highlights the importance of large-scale analysis of the reconnection process. The tail reconnection in Gumics-4 resembles closely the Sweet-Parker model (Laitinen et al., 2005). Analytical considerations show that the Poynting vector divergence in a Sweet-Parker diffusion region is independent of the magnetic Reynolds number, that is, independent of the value or nature of numerical diffusion in the code. This result provides a fixed point for inter-code comparisons and gives credibility to the analysis of numerically induced reconnection.

Methods developed in this paper and by Palmroth et al. (2003) allow us to examine the large-scale reconnection process in the magnetospheric boundaries and in the magnetotail as a function of the driving solar wind and IMF conditions. Palmroth et al. (2006) and Pulkkinen et al. (2006) discuss the energy input into the magnetosphere and conclude that it depends not only on the IMF direction, but also on past values of the IMF; energy input is stronger after southward IMF than it was before southward IMF even if the current driving conditions were identical. This implies that the magnetospheric state is a parameter controlling in part the coupling (and reconnection) process; methods such as developed here suit ideally for analyses of these processes. Analysis of magnetopause reconnection and its dependence on solar wind conditions in Gumics-4 will be the topic of a subsequent article employing methods developed here.

Acknowledgements. The work of T. V. Laitinen was supported by the Magnus Ehrnrooth foundation and that of M. Palmroth by the Academy of Finland. T. I. Pulkkinen thanks the IGPP at the Los Alamos National Laboratory for support during her visit.

Topical Editor I. A. Daglis thanks two referees for their help in evaluating this paper.

\section{References}

Axford, W. I.: Magnetic reconnection, in Magnetic reconnection in space and laboratory plasmas, Geophys. Monogr. Ser., 30, 1, AGU, Washington, D.C., 1984.

Baker, D. N., Pulkkinen, T. I., Angelopoulos, V., Baumjohann, W., and McPherron, R. L.: The neutral line model of substorms: Past results and present view, J. Geophys. Res., 101, 12 975-13010, 1996.

Birn, J., Drake, J. F., Shay, M. A., et al.: Geospace Environmental Modeling (GEM) Magnetic Reconnection Challenge, J. Geophys. Res., 106, 3715-3719, 2001.

Cowley, S. W. H.: A qualitative study of the reconnection between the Earth's magnetic field and an interplanetary field of aribtrary orientation, Radio Sci., 8, 903-913, 1973.

Dorelli, J. C., Hesse, M., Kuznetsova, M. M., Rastaetter, L., and Raeder, J.: A new look at driven magnetic reconnection at the terrestrial subsolar magnetopause, J. Geophys. Res., 109, A12216, doi:10.1029/2004JA010458, 2004.

Dungey, J. W.: Conditions for the occurrence of electrical discharges in astrophysical systems. Phil. Mag., 44, 725-738, 1953

Dungey, J. W.: Interplanetary magnetic field and the auroral zones, Phys. Rev. Lett., 6, 47-48, 1961.

Eriksson, S., Øieroset, M., Baker, D. N., Mouikis, C., Vaivads, A., Dunlop, M. W., Rème, H., Ergun, R. E., and Balogh, A.: Walén and slow-mode shock analyses in the near-Earth magnetotail in connection with a substorm onset on 27 August 2001, J. Geophys. Res., 109, A10212, doi:10.1029/2004JA010534, 2004.

Fedder, J. A., Lyon, J. G., Slinker, S. P., and Mobarry, C. M.: Topological structure of the magnetotail as a function of interplanetary magnetic field direction, J. Geophys. Res., 100, 3613-3622, doi:10.1029/94JA02577, 1995.

Fedder, J. A., Slinker, S. P., Lyon, J. G., and Russell, C. T.: Flux transfer events in global numerical simulations of the magnetosphere. J. Geophys. Res., 107(A5), 1048, doi:10.1029/2001JA000025, 2002.

Hesse, M. and Schindler, K.: A theoretical foundation of general magnetic reconnenction, J. Geophys. Res., 93, 5559-5567, 1988.

Ho, C. M., Tsurutani, B. T., Smith, E. J., and Feldman, W. C.: A detailed examination of a $\mathrm{X}$-line region in the distant tail: ISEE3 observations of jet flow and $B_{z}$ reversals and a pair of slow shocks, Geophys. Res. Lett., 21, 3031-3034, 1994.

Janhunen, P.: GUMICS-3 - A global ionosphere-magnetosphere coupling simulation with high ionospheric resolution, in: Proc. Environmental Modelling for Space-Based Applications, 18-20 September 1996, ESTEC, The Netherlands, ESA SP-392, 1996.

Janhunen, P.: A positive conservative method for magnetohydrodynamics based on HLL and Roe methods, J. Comp. Phys., 160, 649-661, 2000.

Janhunen, P. and Palmroth, M.: Some observational phenomena are well reproduced by our global MHD while others are not: Remarks on what, why and how, Adv. Space Res., 28, 1685-1691, 2001.

Krauss-Varban, D. and Omidi, N.: Large-scale hybrid simulations of the magnetotail during reconnection, Geophys. Res. Lett., 22, 3271-3274, 1995.

Kulsrud, R. M.: A critical review of galactic dynamos, Annual Rev. Astronomy Astrophys., 37, 37-64, 1999.

Laitinen, T. V., Pulkkinen, T. I., Palmroth, M., Janhunen, P., and Koskinen, H. E. J.: The magnetotail reconnection region in a 
global MHD simulation, Ann. Geophys., 23, 3753-3764, 2005, http://www.ann-geophys.net/23/3753/2005/.

LeVeque, R. J.: Numerical methods for conservation laws, Birkhäuser, Basel, 1992.

Øieroset, M., Phan, T. D., Fujimoto, M., Lin, R. P., and Lepping, R. P.: In situ detection of collisionless reconnection in the Earth's magnetotail, Nature, 412, 414-417, 2001.

Palmroth, M., Janhunen, P., Pulkkinen, T., and Peterson, W. K.: Cusp and magnetopause locations in global MHD simulation, J. Geophys. Res., 106, 29 435-29 450, 2001.

Palmroth, M., Pulkkinen, T. I., Janhunen, P., and Wu, C.-C.: Stormtime energy transfer in global MHD simulation, J. Geophys. Res. 108(A1), 1048, doi:101029/2002JA009446, 2003.

Palmroth, M., Janhunen, P., and Pulkkinen, T. I.: Hysteresis in solar wind power input to the magnetosphere, Geophys. Res. Lett., 33, L03107, doi:10.1029/2005GL025188, 2006.

Park, K. S., Ogino, T., and Walker, R. J.: On the importance of antiparallel reconnection when the dipole tilt and IMF $B_{y}$ are nonzero, J. Geophys. Res., 111, A05202, doi:10.1029/2004JA010972, 2006.

Parker, E. N.: Sweet's mechanism for merging magnetic fields in conducting fluids, J. Geophys. Res., 62, 509-520, 1957.

Papadopoulos, K., Goodrich, C., Wiltberger, M., Lopez, R., and Lyon, J. G.: The physics of substorms as revealed by the ISTP, Phys. Chem. Earth C, 24, 189-202, 1999.

Petschek, H. E.: Magnetic field annihilation, in: Physics of Solar Flares, Nasa SP-50, Washington, D.C., USA, 425-439, 1964.

Priest, E. and Forbes, T.: Magnetic Reconnection. MHD Theory and Applications, Cambridge University Press, Cambridge, 2000.

Pulkkinen, T. I., Palmroth, M., Tanskanen, E. I., Janhunen, P., Koskinen, H. E. J., and Laitinen, T. V.: New interpretation of magnetospheric energy circulation, Geophys. Res. Lett., 33, L07101, doi:10.1029/2005GL025457, 2006.
Raeder, J.: Modeling the magnetosphere for northward interplanetary magnetic field: Effects of electrical resistivity, J. Geophys. Res., 104, 17357-17 368, doi:10.1029/JA900159, 1999.

Russell, C. T. and Elphic, R. C.: Initial ISEE magnetometer results - Magnetopause observations, Space Science Rev., 22, 681-715, 1978.

Schindler, K., Hesse, M., and Birn, J.: General magnetic reconnection, parallel electric fields, and helicity, J. Geophys. Res., 93, 5547-5557, 1988.

Siscoe, G. L., Erickson, G. M., Sonnerup, B. U. Ö., Maynard, N. C., Siebert, K. D., Weimer, D. R., and White, W. W.: Global role of $\boldsymbol{E}_{\|}$in magnetopause reconnection: An explicit demonstration, J. Geophys. Res., 106, 13 015-13 022, 2001.

Sonnerup, B. U. Ö., Hasegawa, H., and Paschmann, G.: Anatomy of a flux transfer event seen by Cluster, Geophys. Res. Lett., 31, L11803, doi:10.1029/2004GL020134, 2004.

Sweet, P. A.: The neutral point theory of solar flares, in: Electromagnetic Phenomena in Cosmical Physics, IAU Symp. 6, Cambridge Univ. Press, London, 123-134, 1958.

Vaivads, A., Khotyaintsev, Y., André, M., Retinò, A., and Buchert, S. C.: Structure of the magnetic reconnection diffusion region from four-spacecraft observations, Phys. Rev. Lett., 93, 105 001, doi:10.1103/PhysRevLett.93.105001, 2004.

Vasyliunas, V. M.: Theoretical models of magnetic field line merging, 1., Rev. Geophys. Space Phys., 13, 303-336, 1975.

Walker, R. J., Ogino, T., Raeder, J., and Ashour-Abdalla, M.: A global magnetohydrodynamic simulation of the magnetosphere when the interplanetary magnetic field is southward: the onset of magnetotail reconnection, J. Geophys. Res., 98, 17 235-17 249, 1993. 